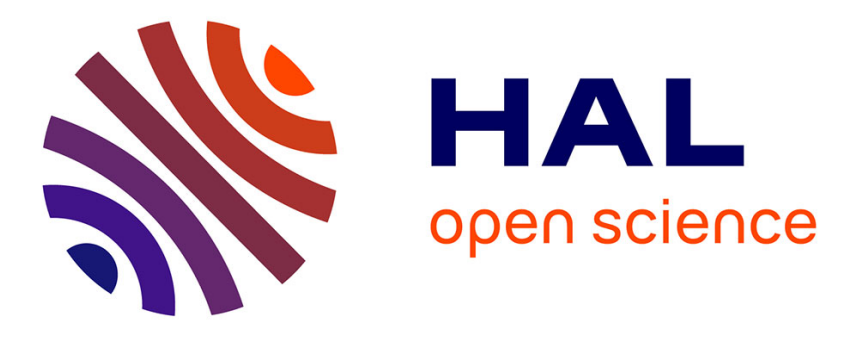

\title{
Polysaccharide capsule and suilysin contribute to extracellular survival of co-cultivated with primary porcine phagocytes
}

Laurentiu Benga, Marcus Fulde, Christina Neis, Ralph Goethe, Peter

Valentin-Weigand

\section{To cite this version:}

Laurentiu Benga, Marcus Fulde, Christina Neis, Ralph Goethe, Peter Valentin-Weigand. Polysaccharide capsule and suilysin contribute to extracellular survival of co-cultivated with primary porcine phagocytes. Veterinary Microbiology, 2008, 132 (1-2), pp.211. 10.1016/j.vetmic.2008.05.005 . hal00532429

\section{HAL Id: hal-00532429 \\ https://hal.science/hal-00532429}

Submitted on 4 Nov 2010

HAL is a multi-disciplinary open access archive for the deposit and dissemination of scientific research documents, whether they are published or not. The documents may come from teaching and research institutions in France or abroad, or from public or private research centers.
L'archive ouverte pluridisciplinaire HAL, est destinée au dépôt et à la diffusion de documents scientifiques de niveau recherche, publiés ou non, émanant des établissements d'enseignement et de recherche français ou étrangers, des laboratoires publics ou privés. 


\section{Accepted Manuscript}

Title: Polysaccharide capsule and suilysin contribute to extracellular survival of Streptococcus suis co-cultivated with primary porcine phagocytes

Authors: Laurentiu Benga, Marcus Fulde, Christina Neis, Ralph Goethe, Peter Valentin-Weigand

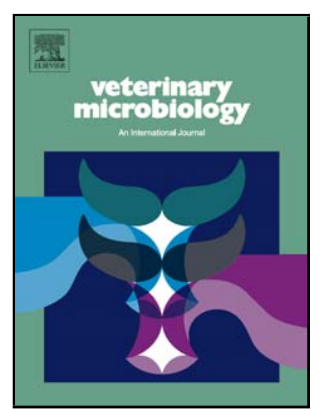

PII: S0378-1135(08)00186-7

DOI: doi:10.1016/j.vetmic.2008.05.005

Reference: VETMIC 4034

To appear in: $\quad$ VETMIC

Received date: $\quad 22-2-2008$

Revised date: 29-4-2008

Accepted date: $\quad$ 5-5-2008

Please cite this article as: Benga, L., Fulde, M., Neis, C., Goethe, R., ValentinWeigand, P., Polysaccharide capsule and suilysin contribute to extracellular survival of Streptococcus suis co-cultivated with primary porcine phagocytes, Veterinary Microbiology (2007), doi:10.1016/j.vetmic.2008.05.005

This is a PDF file of an unedited manuscript that has been accepted for publication. As a service to our customers we are providing this early version of the manuscript. The manuscript will undergo copyediting, typesetting, and review of the resulting proof before it is published in its final form. Please note that during the production process errors may be discovered which could affect the content, and all legal disclaimers that apply to the journal pertain. 
1 Polysaccharide capsule and suilysin contribute to extracellular survival of Streptococcus suis co-

2 cultivated with primary porcine phagocytes

3

4 Laurentiu Benga ${ }^{\S}$, Marcus Fulde ${ }^{\S}$, Christina Neis, Ralph Goethe, Peter Valentin-Weigand*

5

6 Institut für Mikrobiologie, Zentrum für Infektionsmedizin, Stiftung Tierärztliche Hochschule

7 Hannover

8

$9{ }^{\S}$ Both authors contributed equally to this paper

11 *Corresponding author. Mailing address:

12 Stiftung Tierärztliche Hochschule Hannover

13 Zentrum für Infektionsmedizin

14 Institut für Mikrobiologie

15 Bischofsholer Damm 15

1630173 Hannover, GERMANY

17 Phone: $++49-5119537362$

18 Fax: $++49-5119537697$

19 Email: peter.valentin@tiho-hannover.de 


\section{Abstract}

2 Streptococcus suis is a major cause of meningitis, sepsis and arthritis in piglets and a zoonotic agent.

3 Survival in the blood circulation system represents a major step in pathogenesis of S. suis infections.

4 To get further insides into the mechanisms of $S$. suis survival in the host, we compared a highly

5 virulent $S$. suis serotype 2 strain with its non-encapsulated and suilysin-deficient mutants in their

6 abilities to resist phagocytosis and killing by polymorphonuclear neutrophils (PMNs) and

7 mononuclear cells. PMNs displayed a higher capacity to take up encapsulated bacteria than

8 mononuclear cells, whereas both cell types internalised efficiently non-encapsulated $S$. suis.

9 Differentiation of extracellular and intracellular survival of the WT strain revealed that in PMNs the majority of the cell-associated streptococci were intracellular, whereas in mononuclear cells the majority remained attached to the cell surface. S. suis survived mainly extracellularly, since both cells killed intracellular bacteria to a similar extent. As a consequence of different resistance to phagocytosis, only the encapsulated S. suis strains survived co-cultivation with PMNs. Comparison of the WT strain with its encapsulated suilysin-deficient mutant revealed reduced survival of the mutant after co-cultivation with PMNs. Involvement of suilysin in inhibition of phagocytosis was further confirmed by use of anti-suilysin antibodies and recombinant suilysin. Kinetic experiments with PMNs suggested that reduced survival of the mutant strain was mainly associated with an increased uptake, whilst both strains adhered similarly. Concluding, our results indicate that the capsule and the suilysin play important roles in S. suis survival in the host by interfering with phagocytic uptake.

Keywords: Streptococcus suis; capsule; suilysin; phagocytosis. 
1

2

3

\section{Introduction}

S. suis is a major cause of meningitis, sepsis and arthritis in piglets and a zoonotic agent. Bacteria are transmitted via the respiratory route and commonly colonize the palatine tonsils. Under certain, yet unknown, circumstances $S$. suis crosses the respiratory epithelial barrier and disseminates in the blood circulation system. A possible way how S. suis spreads into host tissues might be survival and "travelling" inside (Williams, 1990; Williams and Blakemore, 1990), or closely associated to monocytes and macrophages (Gottschalk and Segura, 2000). Alternatively, S. suis might migrate in blood as free bacteria protected by a thick capsule layer, since a high level of bacteremia usually precedes the onset of bacterial meningitis (Williams, 1990; Tunkel and Scheld, 1993). Nevertheless, experimental evidence supporting either of these theories is limited.

Pathogenicity of many bacteria seems to rely on the protective effect of the capsule against phagocytosis. For S. suis serotype 2 strains, the capsule represents a critical virulence factor, since it is involved in protection against phagocytosis by alveolar macrophages (Charland et al., 1998; Smith et al., 1999). On the other hand, interactions of S. suis with PMNs, is poorly described. These phagocytes participate in the first defense line against $S$. suis infection, however, it is not clear whether they efficiently take up and killed the bacteria (Wibawan and Laemler, 1994; Busque et al., 1998).

Another streptococcal factor probably associated with resistance to phagocytosis is suilysin, a cholesterol dependent cytolysin related to streptolysin O of $S$. pyogenes and pneumolysin of $S$. pneumoniae (Jacobs et al., 1994). These toxins seem to play a multifactorial role in pathogenesis by their cytolytic activities and their inhibition of bactericidal activity of phagocytes (Sierig et al., 2003; Orihuela et al., 2004). However, whether or not suilysin plays a similar role has not been reported yet. To get new insights into $S$. suis survival in the host we compared a highly virulent $S$. suis serotype 2 strain with its non-encapsulated and suilysin-deficient mutants in their abilities to resist phagocytosis and killing by PMNs and mononuclear cells. We found evidence that CPS and suilysin prevent phagocytic uptake, thereby allowing the bacteria to survive extracellularly. 
1

\section{Materials and Methods}

2.1 Bacterial strains and growth conditions. S. suis capsular serotype 2 strain 10 and its noncapsulated isogenic mutant strain $10 \Delta c p s$ EF (designated strain $\Delta c p s$ ) were kindly provided by $\mathrm{H}$. Smith (Lelystad, NL) and have been described in an earlier study (Smith et al., 1999). In addition, a suilysin deficient mutant, strain $\Delta \mathrm{s} l y$, was constructed from strain 10 as described below. The hosts for molecular cloning and protein expression experiments were Escherichia (E.) coli strains DH5a and BL21 (DE 3), respectively. For co-cultivation experiments, S. suis was grown in Todd-Hewitt broth (THB, DIFCO Lab, Detroit, USA) over-night at $37^{\circ} \mathrm{C}$. The next day, an equal volume of fresh THB was then added to the cultures, which were further incubated for 1.5 hours at $37^{\circ} \mathrm{C}$. Then the bacteria were harvested, suspended in PBS ( $\mathrm{pH} 7.3)$ and adjusted photometrically $(600 \mathrm{~nm})$ to a concentration of $2 \times 10^{9}$ bacteria $\mathrm{ml}^{-1}$, before use in further experiments. For some experiments, bacteria were opsonised using either fresh or complement-inactivated $\left(30\right.$ min at $56{ }^{\circ} \mathrm{C}$ ) porcine serum at $20 \%$ in RPMI (final concentration). Serum was obtained from an 8 weeks old piglet which had survived an experimental infection against $S$. suis (Beineke et al., 2008) and was tested positive for the presence of anti-S. suis antibodies by immunoblot as described earlier (Benga et al., 2004a).

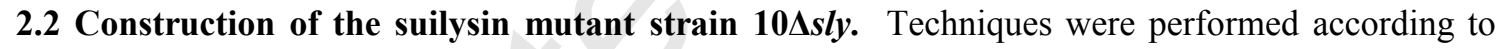
standard procedures (Sambroock and Russel, 2001). Restriction enzymes were purchased from NewEnglandBiolabs (Frankfurt/Main, Germany). To inactivate the sly gene in the strain 10, the gene was interrupted by insertion of an erythromycin cassette. For this, a $1510 \mathrm{bp}$ fragment of the sly gene was amplified by PCR using the primer pair SL4-L (AATTCCATATGAGAAAAAGTCGCACTTGATTT) and SL4-R (CGGGATCCTTACTCTATCACCTCATCCGCATAC). Subsequently, the product was cloned into the plasmid pCRII-TOPO (Invitrogen), then the fragment was digested with SacI-PstI and ligated into the plasmid pBluescript II SK(+) (Stratagene, Amsterdam, The Netherlands) digested with the same enzymes. The erythromycin cassette derived from pICerm (kindly provided by C. Baums) was digested with $P v u \mathrm{II}$ and ligated into the $H p a \mathrm{I}$ site of the sly gene to create pBlue/sly/erm. The last plasmid was electroporated $(2.5 \mathrm{kV}, 200 \Omega, 25 \mu \mathrm{F})$ into S. suis strain 10 . Transformants which had undergone double-crossover events were genotipically confirmed using PCR and Southern Blot 
1 analysis. The phenotype of the suilysin mutant was confirmed by a hemolysis assay, as described by

2 Takamatsu et al. (2001).

\subsection{Cloning, expression, and purification of recombinant suilysin and production of polyclonal} anti-suilysin antibodies. The suilysin gene coding region of $S$. suis strain 10 was amplified by PCR using the primers slyAgefor (TGTACCGGTGATTCCAAACAAGATATTAA) and slyAgerev (CCAACCGGTCTCTATCACCTCATCCGCAT). The PCR product was digested using the enzyme AgeI and inserted into the vector pET45b (Novagen, Darmstadt, Germany), which was also digested by AgeI. The resulting vector pET45bsly, was amplified in E. coli DH5- $\alpha$ cells, then purified and sequenced using the primers pET45b5forward (AGGATCGAGATCGATCTCG), pET45b5reverse (TTCTTCTTTTCTCTGCGA) and pET45b (CCTGCAGGCGCGCCGTGTAC). Recombinant suilysin was expressed in E. coli BL21 (DE3) cells after induction with isopropyl- $\beta$-D-thiogalactoside and was purified according to ,purification of polyhistidine-tagged proteins from E. coli under denaturating conditions" with Protino ${ }^{\circledR}$ Ni-TED 2000 packed columns (Macherey-Nagel, Dueren, Germany). The eluted protein was renaturated by dialysis against $25 \mathrm{mM}$ Tris- $\mathrm{HCl}$ with decreasing urea concentrations. The last dialysis step was performed against a buffer containing $25 \mathrm{mM}$ Tris- $\mathrm{HCl}$ (pH 7.0), $50 \mathrm{mM} \mathrm{NaCl}, 1 \mathrm{mM}$ EDTA, $5 \%$ (v/v) Glycerol. Protein concentration was measured according to 'microplate assay protocol' (Bio-Rad), and the haemolytic activity was tested as described as described by Takamatsu et al. (2001). The protein was stored at $-20^{\circ} \mathrm{C}$ after adding $5 \mathrm{mM}$ dithiothreitol. Polyclonal rabbit anti-rSLY antibodies were raised by immunisations of a New Zealand white rabbits using the purified protein.

2.4. Isolation of porcine phagocytes. Freshly collected heparinised blood form healthy piglets was mixed with an equal volume of $0.87 \% \mathrm{NaCl}$, then layered on Ficoll-Hypaque 1077 (Biochrom, Berlin, Germany) and subsequently centrifuged for $30 \mathrm{~min}$ at $400 \mathrm{~g}$ and $20^{\circ} \mathrm{C}$. Centrifugation resulted in formation of two fractions, an interface containing mononuclear cells, and a pellet containing PMNs and erythrocytes. Following, the erythrocytes were removed by hypotonic lysis, and the purified PMNs were suspended in RPMI medium. The mononuclear fraction was harvested from the interface, washed twice with $0.87 \% \mathrm{NaCl}$ and then suspended in RPMI. The viability of the cells was $>95 \%$ as 
1 determined by trypan blue exclusion assay. The purified cell fractions were immediately used for

2 phagocytosis and killing assays.

3 2.5. PMNs killing assay. This was performed as described by Wessels et al.(1991). Briefly, $400 \mu 1$ leukocyte suspension $\left(10^{7}\right.$ cells $\left./ \mathrm{ml}\right)$ was mixed 1:1 with $S$. suis in PBS, either alone, or in the presence of $100 \mu \mathrm{l}$ porcine serum (fresh or complement-inactivated, $30 \mathrm{~min}$ at $56^{\circ} \mathrm{C}$ ) and incubated at $37^{\circ} \mathrm{C}$ under continuous end-over-end rotation ( $6 \mathrm{rpm} / \mathrm{min})$. In some experiments, anti-suilysin polyclonal antiserum or pre-immune rabbit serum, or rSLY were included. Aliquots of $20 \mu \mathrm{l}$ were plated immediately and at the end of experiments $(1 \mathrm{~h})$. Samples containing only cells or bacteria were used as controls. Results were expressed as log fold change in CFU.

\subsection{Determination of adherence, phagocytosis and intracellular survival by antibiotic protection}

assay. This was performed as described by Smith et al. (1999) with some modifications. Briefly, porcine phagocytes (PMNs, mononuclear cells) $\left(10^{7} / \mathrm{ml}\right)$ were mixed with equal numbers of preopsonised bacteria (MOI 1:1) in a final volume of $2 \mathrm{ml}$ and incubated for $15 \mathrm{~min}$ at $37^{\circ} \mathrm{C}$ under continuous rotation to allow phagocytosis. To remove bacteria not associated with the cells phagocytes were washed twice with ice-cold RPMI and resuspended in the initial volume of medium. Then, parallel aliquots of $400 \mu 1$ were harvested either immediately $(t=0)$ or after further incubation for 45 $\min$ at $37^{\circ} \mathrm{C}(\mathrm{t}=45)$. These were treated either with $100 \mu \mathrm{g}$ gentamicin and $5 \mu \mathrm{g}$ penicillin $\mathrm{ml}^{-1}$ to kill extracellular adherent $S$. suis and quantify the intracellular bacteria, or they were incubated further in RPMI without antibiotics to quantify the total number of intra- and extracellular $S$. suis for 45 min at $37^{\circ} \mathrm{C}$. The number of extracellular bacteria was determined by subtracting the intracellular bacteria from the total number of intra- and extracellular bacteria. In some experiments, to determine the phagocytic and intracellular killing ability of PMNs, samples were taken immediately $(t=0)$, and after $60 \mathrm{~min}(\mathrm{t}=60)$ and treated with antibiotics for $45 \mathrm{~min}$ at $4^{\circ} \mathrm{C}\left(\right.$ instead of $\left.37^{\circ} \mathrm{C}\right)$ in order to exclude additional killing of intracellular bacteria by neutrophils during antibiotic killing of extracellular bacteria. The efficiency of antibiotic killing was proven by platings of the supernatants. To determine the adherence of $S$. suis to PMNs, parallel aliquots of phagocytes were treated with 1 $\mu \mathrm{g} / \mathrm{ml}$ cytochalasin D (Calbiochem, Darmstadt, Germany) 30 min prior to infection, as well as $15 \mathrm{~min}$ after the infection to inhibit the phagocytic uptake. Subsequently, the samples were washed twice, and 
1 the phagocytic cells were lysed for $15 \mathrm{~min}$ at room temperature in $400 \mu 1$ of $1 \%$ saponin. The number

2 of CFU in the suspensions was determined by platings on THB agar.

\subsection{Determination of adherence and phagocytosis by double immunofluorescence microscop $y$.} Approximately $10^{7}$ pre-opsonised bacteria were added to $\sim 10^{6}$ phagocytes (PMNs, mononuclear cells) in $500 \mu \mathrm{RPMI}$ and incubated for $1.5 \mathrm{~h}$ at $37^{\circ} \mathrm{C}$ using end-over-end rotation. Then the phagocytes were allowed to adhere for $1 \mathrm{~h}$ to glass coverslips placed in 24 well plates. Subsequently, the wells were washed, fixed with $3.7 \%$ formaldehyde and examined by differential immuno-fluorescence (DIF) for intra- and extracellular bacteria as previously described (Benga et al., 2004b).

2.8 Cell cytotoxicity assays. Trypan blue exclusion assay $(0.2 \%$ solution $)$ and lactate dehydrogenase (LDH) release assays were used to assess a possible damage of porcine phagocytes during cocultivation with S. suis as described previously (Benga et al., 2004b)

2.9 Statistical analysis. Experiments were performed at least 3 times, and results were expressed as means $+/$ - standard deviation (SD). Data were analyzed by the Wilcoxon test. A $P$ value $<0.05$ was considered significant.

\section{Results}

\subsection{Extra- and intracellular survival of $S$. suis after co-cultivation with PMNs and mononuclear}

cells. To determine the contribution of different phagocytic cell types to opsonophagocytic killing of S. suis, we assessed the ability of the virulent encapsulated S. suis strain 10 to resist phagocytosis and killing by porcine PMNs and mononuclear cells. PMNs displayed a higher capacity to phagocytose encapsulated S. suis than mononuclear leukocytes (fig. 1). After 45 min, approximately $67 \%$ of the PMN-associated bacteria were detected intracellularly, whereas approx. $75 \%$ of bacteria associated with the mononuclear cells were found extracellularly. After further incubation for $45 \mathrm{~min}$, approx. $80 \%$ of the internalized bacteria were killed, whereas the number of extracellular bacteria increased by approx. $70 \%$, independent of the phagocyte cell type (fig. 1). To confirm these findings we used DIF to distinguish between intra- and extracellular bacteria. In correlation with the plating results, PMNs phagocytosed efficiently encapsulated $S$. suis, i.e. numerous intracellular bacteria were detected and only few adherent bacteria were seen. In contrast, mononuclear cells were significantly less effective 
1 in phagocytosis, i.e. only few intracellular bacteria were detected (fig. 1). The non-encapsulated strain

$2 \Delta c p s$ was efficiently taken up by both phagocytes (data not shown).

$3 \quad 3.2$ Role of suilysin and capsule in $S$. suis survival after co-cultivation with PMNs. The relevance of suilysin for survival after co-cultivation with PMNs was evaluated by comparing the strains 10 and its suilysin-deficient mutant $\Delta s l y$. The non-encapsulated mutant $\Delta c p s$ was included as a phagocytosis sensitive control.

Although PMNs phagocytosed and killed the wt strain to a certain extent, as described above, it seemed that phagocytosis and killing rates were not efficient to eliminate all bacteria, since wild-type (wt) S. suis survived co-cultivation with isolated PMNs (fig. 2). Moreover, it was able to multiply, in contrast to strain $\Delta c p s$, which was killed very efficiently. Interestingly, no difference in survival of the wt strain was seen after opsonisation. On the contrary, opsonisation of the non-encapsulated mutant strain resulted in a further increase of killing in comparison with non-opsonic conditions (fig. 2). After opsonisation with heat-inactivated serum this strain was killed as efficiently as with fresh serum (data not shown). Strain $\Delta s l y$ survived at intermediate levels in comparison with the wt and strain $\Delta c p s$ when co-cultivated with isolated porcine PMNs (fig. 2). Similar to strain 10, strain $\Delta s l y$ was able to multiply and no difference was seen after opsonisation, in contrast to strain $\Delta c p s$. However, the suilysin mutant multiplied to significantly lower extent than the wild type when co-cultivated with PMNs.

To assess the possibility that the increased survival of the wt strain as compared to strain $\Delta s l y$ was due to suilysin-mediated cytotoxicity, we evaluated the cell viability at the end of the experiments by trypan blue staining and measurement of LDH release in the supernatant. Both tests revealed that all strains were not cytotoxic under our experimental conditions (data not shown),. Moreover, all three strains grew equally well in the control assay without cells. To further confirm the role of suilysin in the protection of S. suis against PMNs, we produced recombinant suilysin and raised rabbit polyclonal antiserum against it. In the PMNs killing assay, we observed that treatment with anti-suilysin antibodies lead a decreased survival of the wt strain 10 (fig. 3A). On the other hand, complementation of strain $\Delta s l y$ with recombinant suilysin at sublystic concentrations resulted in an increased growth capacity in the presence of porcine PMNs restoring the wt phenotype (fig. 3B). 
1 3.3. Suilysin mediates inhibition of the phagocytic uptake by PMNs. In order to see whether the

2 differences in survival of the 3 tested strains were related to resistance either to phagocytosis or to intracellular killing, we quantified the ability of porcine PMNs to take up and kill the wt strain, strain $\Delta s l y$ and strain $\Delta c p s$ in a modified antibiotic protection assay. Phagocytic uptake was recorded after 15 min of co-cultivation $(\mathrm{t}=0)$, and bacterial resistance to intracellular killing was determined after further incubation for $60 \mathrm{~min}$. To avoid possible killing of ingested bacteria by PMNs during antibiotic treatments, these were done at $4^{\circ} \mathrm{C}$. As expected, we found a significantly higher extent of internalization in strain $\Delta c p s$ in comparison with the encapsulated strains at $\mathrm{t}=0$ (fig. $4 \mathrm{~A})$. Moreover, strain $\Delta$ sly was significantly better internalized than the wt strain. After $60 \mathrm{~min}$, the percentage of intracellular bacteria decreased substantially in strain $\Delta c p s$ but to a lesser extent in encapsulated strains (fig. 4A). To exclude the possibility that the increased internalization of the strain $\Delta s l y$ compared to the wild-type was due to an increased ability to adhere to the PMNs, we assessed in parallel samples the adherence capacity of the three tested S. suis strains after inhibition of phagocytic uptake by cytochalasin D. Both encapsulated strains adhered equally well to the PMNs (fig. 4B).

\section{Discussion}

After invasion into deeper tissues, dissemination in the blood circulation is a crucial step in establishment of S. suis infection. As postulated previously (Gottschalk and Segura, 2000), S. suis might be found in blood either as free bacteria or in association with blood cells, i. e. monocytes. In the latter case, the bacteria might "travel" either intracellularly (Williams, 1990; Williams and Blakemore, 1990) or closely bound to the cell surface (Gottschalk and Segura, 2000). The published reports supporting either of the two theories provided some evidence that $S$. suis survives cocultivation with porcine phagocytes (Smith et al., 1999; Chabot-Roy et al., 2006). At present, however, it is not clear how S. suis survives killing by phagocytes, and to what extent different phagocytes (PMNs, mononuclear cells) and bacterial factors contribute to this process.

We designed experiments that allowed further evaluation of $S$. suis-phagocyte interactions. Our results indicated that PMNs and mononuclear cells differed significantly in their capacity to internalize encapsulated S. suis. PMNs phagocytosed encapsulated S. suis, whereas mononuclear cells 
1 internalized the bacteria very poorly. On the other hand, both cell types phagocytosed efficiently non-

2 encapsulated strains. Once internalized, even encapsulated $S$. suis were easily killed by both

3 phagocytes. However, in the case of encapsulated S. suis the internalization rate seemed not to be

4 sufficient to prevent infection, since encapsulated S. suis were able to multiply extracellularly after co-

5 cultivation with phagocytes. Our results suggest that the capacity of the bacteria to avoid efficient

6 phagocytic uptake contributed to their survival, and that S. suis survived closely attached to the cell

7 surface.

8 We further showed that encapsulated wt strain and strain $\Delta s l y$, but not $\Delta c p s$ survived phagocytic

9 clearance when co-cultivated with PMNs independent of opsonisation, further supporting the important role of the capsule as an antiphagocytic resistance factor (Charland et al., 1998; Smith et al., 1999). Opsonisation substantially increased killing by PMNs of the non-encapsulated mutant $\Delta c p s$ (which was killed also at non-opsonic condition), whereas the encapsulated bacteria survived cocultivation by leukocytes even after opsonisation. There was no difference in killing of the capsule mutant when complement deprived serum was used, suggesting that complement mediated opsonisation did not play an essential role, similar to what has been described for group B streptococci (Valentin-Weigand et al., 1996). Nevertheless, the precise role of the complement system in virulence of S. suis remains to be elucidated.

In other pathogenic streptococci the cholesterol binding toxins have been shown to be involved in pyogenes by PMNs in vitro and enhanced their virulence in vivo in a mouse infection model (Sierig et al., 2003). And in pneumococci, Orihuela et al. (2004) have elegantly demonstrated the multifactorial role of the pneumolysin in pathogenesis. In our study we demonstrated that suilysin contributed to bacterial survival in the presence of porcine phagocytes in vitro, in accordance to a recently published study (Chabot-Roy et al., 2006). Moreover, we were able to show that anti-suilysin antibodies could neutralize these effects and that complementation with recombinant suilysin restored the wt phenotype. Since we performed our experiments at subcytolytic conditions, cytotoxic effects on phagocytes could be excluded. Rather, an adherence-dependent action of the toxin seems to be the 
1 mechanism involved in survival. Based on our findings we assumed that beside the capsule, suilysin

2 contributed to extracellular survival of S. suis after cocultivation with PMNs.

3 Recently, it has been shown that streptolysin $\mathrm{O}$ prevents internalisation of $S$. pyogenes by ( (6 7 oropharyngeal keratinocytes (Hakansson et al., 2005). Moreover, this toxin mediated evasion of lysosomal uptake (Hakansson et al., 2005), thus facilitating the survival of bacteria localized mainly extracellularly. Consistent with this, we demonstrated that the suilysin-deficient mutant $\Delta s l y$ was taken up significantly better than the wild type strain, whereas the adherence rates of the two strains were similar. This suggests that suilysin is involved in bacterial dissemination in the blood circulation. Indeed, very recently it was shown that the expression of the sly gene was up-regulated in blood (Tan et al., 2008). Earlier studies showed that a sly mutant was attenuated in vivo in a murine (Allen et al., 2001) but not a porcine (Lun et al., 2003) infection model, which may be explained by the differences between the mouse and porcine infection models for S. suis (Vecht et al., 1997).

Taken together, depending on the route and stage of infection, suilysin may well contribute to virulence by interfering with phagocytosis, especially in low-encapsulated $S$. suis strains. This is supported by the fact that suilysin expressing capsule-deficient $S$. suis strains could be isolated from invasive locations in pigs (Allgaier et al., 2001; Benga et al., 2004b). Future studies will have to address the molecular mechanisms of suilysin-mediated effects on host cells, and will help to dissect the possible interplays of the different virulence-associated factors of $S$. suis in infection.

\section{Acknowledgements}

We gratefully acknowledge the Klinik fuer Klauentiere of the Stiftung Tieraerzliche Hochschule Hannover for providing porcine blood. We thank Hilde Smith, Lelystad, NL, for kindly providing $S$. suis strains, and Christoph Baums for his help in construction of the suilysin mutant. This study was supported by the Deutsche Forschungsgemeinschaft (DFG), Bonn, Germany (SFB587 and GRK745). 
1

2

\section{References}

Allen, A.G., Bolitho, S., Lindsay, H., Khan, S., Bryant, C., Norton, P., Ward, P., Leight, J., Morgan, J., Riches, H., Eastty, S., Maskell, D., 2001. Generation and characterisation of a defined mutant of Streptococcus suis lacking suilysin. Infect. Immun. 69, 2732-2735

Allgaier, A., Goethe, R., Wisselink, H.J., Smith, H.E., Valentin-Weigand, P., 2001. Relatedness of Streptococcus suis isolated of various serotypes and clinical backgrounds as evaluated by macrorestriction analysis and expression of potential virulence traits. J. Clin. Microbiol. 39, 445-453.

Beineke, A., Bennecke, K., Neis, C., Schröder, C., Waldmann, KH., Baumgärtner, W., Valentin-Weigand, P., Baums, C.G., 2008. Comparative evaluation of virulence and pathology of Streptococcus suis serotypes 2 and 9 in experimentally infected growers. Vet Microbiol. 128, 423-430.

Benga, L., Goethe, R., Grosse-Beilage, E., Valentin-Weigand, P., 2004a. Immunogenicity of murein-associated proteins from temperature stressed Streptococcus suis cultures. J. Vet. Med. B. 51, 272-277.

Benga, L., Goethe, R., Rohde, M., Valentin-Weigand, P., 2004b. Non-encapsulated strains reveal novel insights in invasion and survival of Streptococcus suis in epithelial cells. Cell. Microbiol. 6, 867-881.

Busque, P., Higgins, R., Senechal, S., Marchand, R., Quessy S., 1998. Simultaneous flowcytometric measurement of Streptococcus suis phagocytosis by polymorphonuclear and mononuclear blood leukocytes. Vet. Microbiol. 63, 229-238.

Chabot-Roy, G., Willson, P., Segura, M., Lacouture, S, Gottschalk, M., 2006. Phagocytosis and killing of Streptococcus suis by porcine neutrophils. Microb. Pathog. 41, 21-32.

Charland, N., Harel, J., Kobisch, M., Lacasse, S., Gottschalk, M., 1998. Streptococcus suis serotype 2 deficient in capsular expression. Microbiol. 144, 325-332.

Gottschalk, M., Segura, M., 2000. The pathogenesis of the meningitis caused by Streptococcus suis: the unresolved questions. Vet. Microbiol. 76, 259-272.

Hakansson, A., Bentley, C.C., Shakhnovic, E.A., Wessels, M.R., 2005. Cytolysin-dependent evasion of lysosomal killing. P.N.A.S. 102, 5192-5197.

Jacobs, A.A., Loeffen, P., van der Berg, A., Storm, P., 1994. Identification, purification and characterisation of a thiol-activated hemolysin (suilysin) of Streptococcus suis. Infect. Immun. 62, 1742-1748.

Lun, S., Perez-Casal, J., Connor, W., Wilsson, P.J., 2003. Role of suilysin in pathogenesis of Streptococcus suis capsular serotype 2. Microb. Pathogen. 34, 27-37.

Orihuela, C.J., Gao, G., Francis, K.P., Yu, J., Tuomanen, E.I., 2004. Tissue-specific contributions of pneumococcal virulence factors to pathogenesis. J. Inf. Dis. 190, 1661-1669. 
1 Sambroock, J., Russel, D.W., 2001. Molecular Cloning. Cold Spring Harbor Laboratory Press, New York.

2 Sierig, G., Cywes, C., Wessels, M.R., Ashbaugh, C.D., 2003. Cytotoxic effects of streptolysin O and streptolysin

3 S enhance the virulence of poorly encapsulated group A streptococci. Infect. Immun. 71, 446-455.

4 Smith, H.E., Damman, M., van der Velde, J., Wagennar, F., Wisselink, H.J., Stockhofe-Zurvieden, N., Smits,

5 M.A., 1999. Identification and characterisation of the cps locus of Streptococcus suis serotype 2: the capsule

6 protects against phagocytosis and is an important virulence factor. Infect. Immun. 67, 1750-1756.

7 Takamatsu, D., Osaki, M., Sekizaki, T., 2001. Thermosensitive suicide vectors for gene replacement in

$8 \quad$ Streptococcus suis. Plasmid 46, 140-148.

9 Tan, C., Liu, M., Jin M., Liu, J., Chen, Y., Wu., T, Fu, T., Bei, W., Chen, H., 2008. The key virulence-associated 10 genes of Streptococcus suis type 2 are upregulated and differentially expressed in vivo. FEMS Microbiol. Lett. $278,108-14$

Tunkel, A.R., Scheld, W.M., 1993. Pathogenesis and pathophysiology of bacterial meningitis. Clin. Microbiol. Rev. 6, 118-136.

Valentin-Weigand, P., Benkel, P., Rohde, M., Chhatwal, G.S., 1996. Entry and intracellular survival of group B streptococci in J774 macrophages. Infect. Immun. 64, 2467-2473.

Vecht, U., Stockhofe-Zurwieden, N., Tetenburg, B.J., Wisselink, H.J., Smith, H.E., 1997. Virulence of Streptococcus suis type 2 for mice and pigs appeared host-specific. Vet. Microbiol. 58, 53-60.

Wessels, M.R., Moses, A.E., Goldberg, J.B., DiCesare, T.J., 1991. Hyaluronic acid capsule is a virulence factor for mucoid group A streptococci. Proc. Natl. Acad. Sci. 88, 8317-8321.

Wibawan, I.W.T., Lämmler, C., 1994. Relation between encapsulation and various properties of Streptococcus suis. J. Vet. Med. 41, 453-459.

Williams, A.E., 1990. Relationship between intracellular survival in macrophages and pathogenicity of Streptococcus suis type 2 isolates. Microb. Pathog. 8, 189-196.

Williams, A.E., Blakemore, W.F., 1990. Pathogenesis of meningitis caused by Streptococcus suis type 2. J. Inf. Dis. $162,474-481$. 


\section{$1 \quad$ Figure legends}

\section{$2 \quad$ Figure 1}

3 Kinetics of adherence, phagocytosis and killing of S. suis strain 10 by porcine PMNs (upper graph) and

Figure 2

Contribution of capsule and suilysin to survival of $S$. suis to co-cultivation by porcine PMNs. S. suis strains 10 , $\Delta s l y$ and $\Delta c p s$ were incubated with PMNs, either alone (non-opsonised) or in presence of $20 \%$ porcine serum. CFU of surviving bacteria were quantified by platings at the beginning and at the end of the experiment. Data are expressed as mean log fold change in CFU after $1 \mathrm{~h}$. Means and SD of five independent experiments are shown. *, $P$ values $<0.05$.

\section{Figure 3}

Neutralization (A) and complementation (B) of the suilysin in the S. suis WT and suilysin mutant respectively. For this, S. suis strains 10 and $\Delta s l y$ were incubated with PMNs for $1 \mathrm{~h}$ at $37^{\circ} \mathrm{C}$, in the presence of $20 \%$ antisuilysin serum or preimmune serum (A) or in the presence of rSly (10 $\mu \mathrm{g} / \mathrm{ml})$ (B). CFU of surviving bacteria were quantified by platings at the beginning and the end of the experiment. Data are expressed as \% survival of the fold change in CFU after $1 \mathrm{~h}$ achieved by the controls. Means and SD of five independent experiments are shown. *, $P$ values $<0.05$.

\section{Figure 4}

Contribution of suilysin and capsule to adherence (B), phagocytosis and killing (A) by porcine PMNs. S. suis strains 10, $\Delta$ sly and $\Delta c p s$ were opsonised with $20 \%$ porcine immune serum, then co-cultivated with PMNs for 15 min at $37^{\circ} \mathrm{C}$ to allow phagocytosis. Subsequently, the phagocytes were washed and the number of intracellular bacteria was determined by platings after 0 and $60 \mathrm{~min}$ of further incubation. To determine the adherence parallel aliquots of PMNs were treated with cytochalasin D to inhibit the cytoskeleton mediated uptake. The total number of adherent and intra-cellular CFU in the suspensions was quantified by platings. Results were expressed 
1 as $\%$ adherence or invasion (\% recovered CFU of the inoculum). Means and SD of five independent experiments 2 are shown. *, $P$ values $<0.05$. 


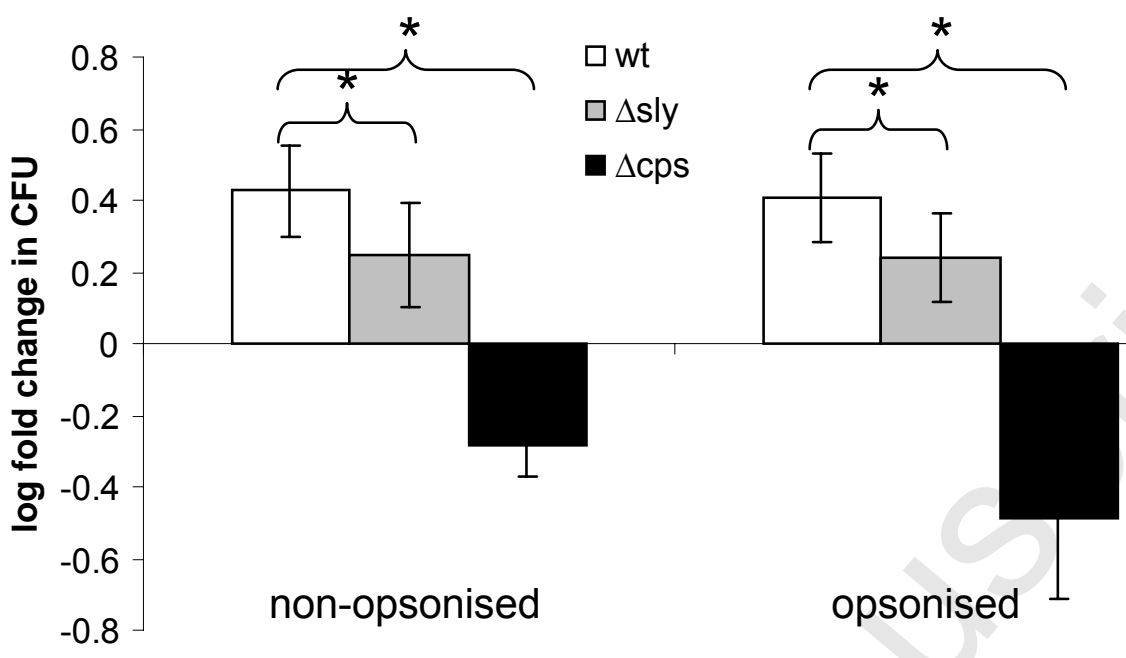


A

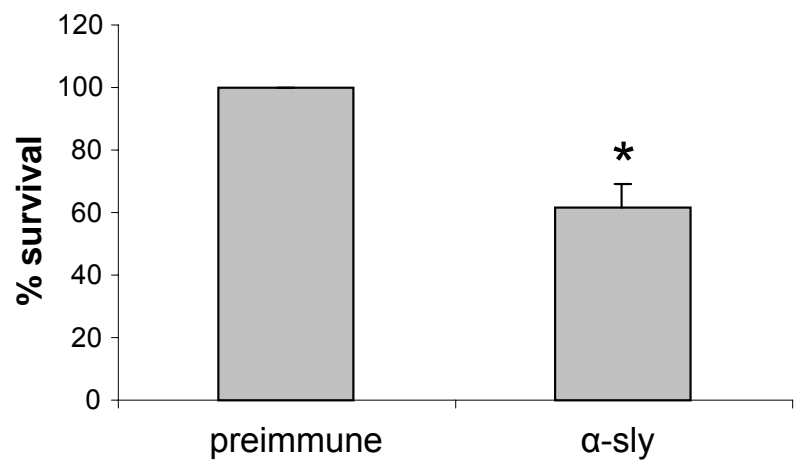

B

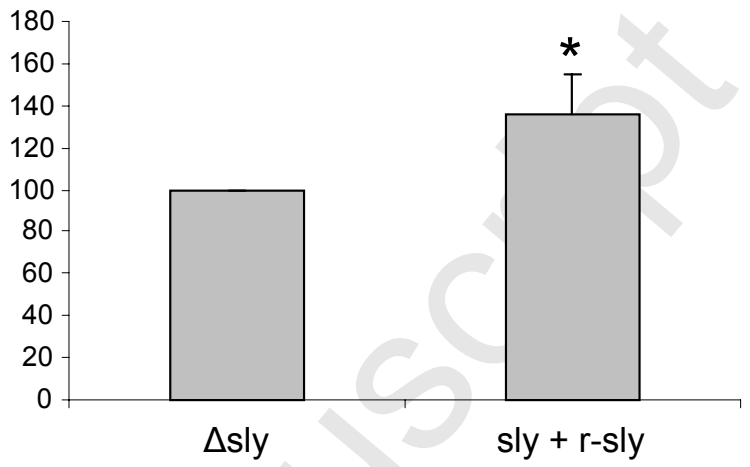



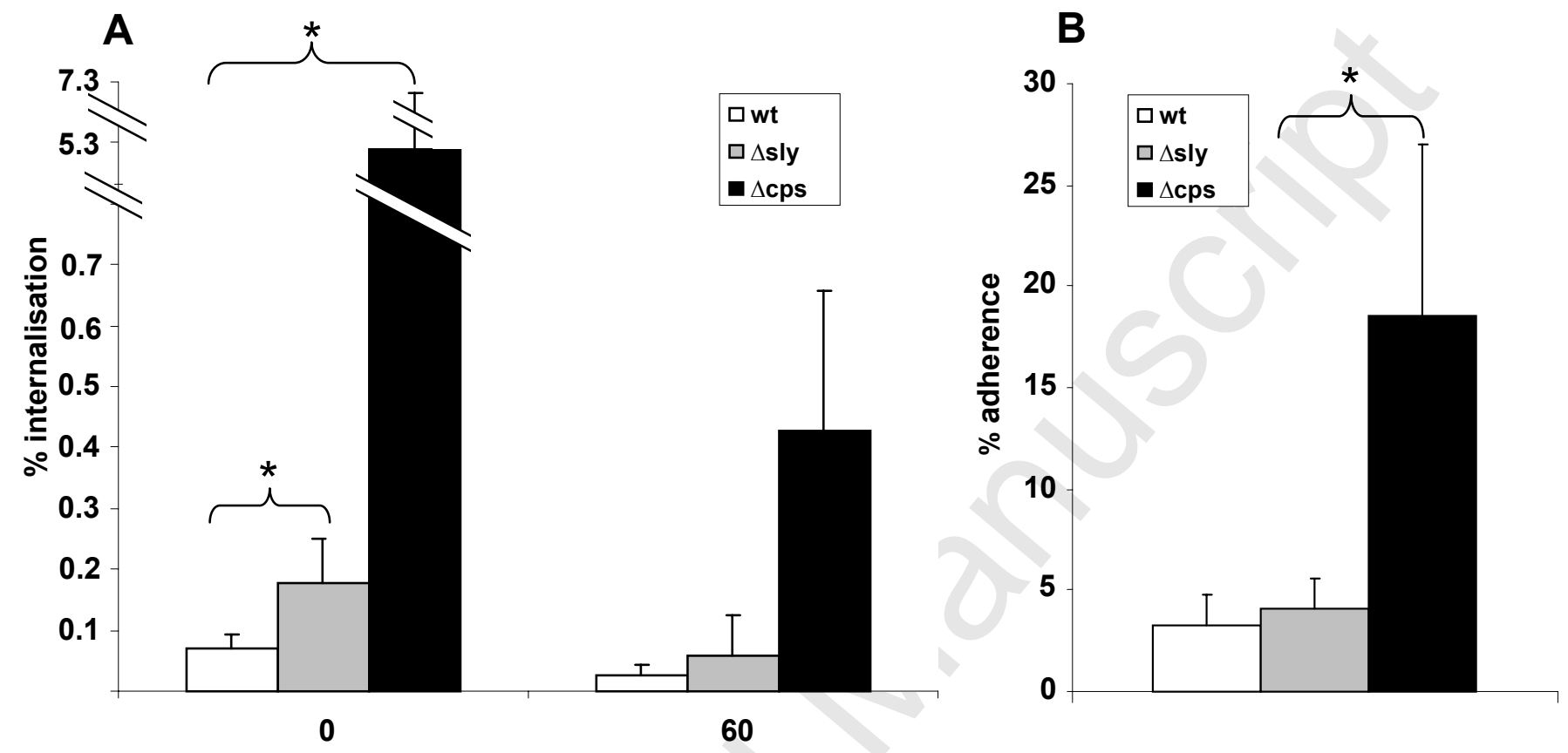